\section{Comparison of Intensity Modulated Radiotherapy Treatment Plans Between I.5T MR-Linac and Conventional Linac}

\author{
Shouliang Ding, MS', Yongbao Li, PhD', Hongdong Liu, PhD', \\ Rui Li, PhD', Bin Wang, PhD', Jun Zhang, PhD', Yan Chen, PhD', \\ and Xiaoyan Huang, $\mathbf{M S}^{\prime}{ }^{\prime}$
}

Technology in Cancer Research \& Treatment

Volume 20: I-II

(C) The Author(s) 2021

Article reuse guidelines:

sagepub.com/journals-permissions DOI: | 0.1 | 177/| 53303382098587| journals.sagepub.com/home/tct

(S)AGE

\begin{abstract}
In this study, we assess the dosimetric qualities and usability of planning for I.5 T MR-Linac based intensity modulated radiotherapy (MRL-IMRT) for various clinical sites in comparison with IMRT plans using a conventional linac. In total of 30 patients with disease sites in the brain, esophagus, lung, rectum and vertebra were re-planned retrospectively for simulated MRL-IMRT using the Elekta Unity dedicated treatment planning system (TPS) Monaco (v5.40.0I). Currently, the step-and-shoot (ss) is the only delivery technique for IMRT available on Unity. All patients were treated on an Elekta Versa HD ${ }^{T M}$ with IMRT using the dynamic multileaf collimator (dMLC) technique, and the plans were designed using Monaco v5. I I. For comparison, the same dMLC-IMRT plan was recalculated with the same machine and TPS but only changing the technique to step-and-shoot. The dosimetric qualities of the MRL-IMRT plans, to be evaluated by the Dose Volume Histograms (DVH) metrics, Homogeneity Index and Conformality Index, were compared with the clinical plans. The planning usability was measured by the optimization time and the number of Monitor Units (MUs). Comparing MRL-IMRT with conventional linac based plans, all created plans were clinically equivalent to current clinical practice. However, MRL-IMRT plans had higher dose to skin and larger low dose region of normal tissues. Furthermore, MRL-IMRT plans had significantly reduced optimization time by comparing conventional linac based plans. The number of MUs of MRL-IMRT was increased by $23 \%$ compared with ss-IMRT, and no difference from dMLC-IMRT. In conclusion, clinically acceptable plans can be achieved with I.5 T MR-Linac system for multiple tumor sites. Given the differences in machine characteristics, some minor differences in plan quality were found between MR-Linac plans and current clinical practice and this should be considered in clinical practice.
\end{abstract}

\title{
Keywords
}

MR-linac, intensity modulated radiotherapy, treatment planning, plan evaluation, MRI-guided radiotherapy

\begin{abstract}
Abbreviations
MRI, Magnetic Resonance Imaging; CBCT, Cone Beam Computed Tomography; ERE, Electron Return Effect; MLC, Multi-Leaf Collimator; IMRT, Intensity Modulated Radiotherapy; dMLC, dynamic Multi-Leaf Collimator; ss, step and shoot; DVH, Dose-volume histograms; HI, Homogeneity index; $\mathrm{Cl}$, Conformality index; OAR, Organs at risk
\end{abstract}

Received: July 10, 2020; Revised: November 05, 2020; Accepted: December 14, 2020.

\section{Introduction}

Magnetic resonance image (MRI) guided radiotherapy can provide high and versatile soft-tissue contrast imaging for realtime plan adaptation and target position monitoring during irradiation. ${ }^{1}$ Improvements in soft tissue contrast are desirable for a number of treatment sites, for example pelvis or abdominal tumors, where the Cone Beam CT (CBCT) imaging has poor performance. ${ }^{2}$ To enable MR imaging prior to and during

\footnotetext{
' State Key Laboratory of Oncology in South China, Collaborative Innovation Center for Cancer Medicine, Sun Yat-sen University Cancer Center, Guangzhou, China

${ }^{2}$ Elekta (Shanghai) Instrument Ltd, China
}

Corresponding Author:

Xiaoyan Huang, State Key Laboratory of Oncology in South China, Collaborative Innovation Center for Cancer Medicine, Sun Yat-sen University Cancer Center, Guangzhou, Guangdong 510060, China.

Email: huangxiaoy@sysucc.org.cn 
treatment, the MR-Linac (Elekta, AB, Stockholm, Sweden) from the MR-Linac Consortium has combined a 1.5 T Philips scanner (Best, The Netherlands) and a 7 MV flattening filter free (FFF) linac. ${ }^{3}$ This system allows for daily MR image guidance and real-time imaging throughout the treatment fraction, which is ideal for managing and monitoring both interfraction and intrafraction motion, without incurring additional radiation exposure. ${ }^{4-6}$

The MR-Linac is significantly different from the conventional linac in many aspects. An important feature is the presence of a magnetic field which inevitably affects radiation dose distribution. Previous studies using Monte Carlo calculations on phantoms reported that the magnetic field can lead to asymmetric point spread kernels, resulting in reduced build-up regions and asymmetric beam penumbra regions which are also shifted. ${ }^{7}$ The magnetic field will change the paths of secondary electrons in tissue, particularly at the tissue-air and tissue-lung interfaces. For example, exiting electrons at interface will be forced back into the tissue under the Lorentz force, which termed as Electron Return Effect (ERE), and this can lead to significantly different dose distribution at the interface from that of a conventional linac. ${ }^{8}$ The presence of a $1.5 \mathrm{~T}$ high magnetic field (B-field) in the MR-Linac during beam delivery will cause the ERE at tissue interfaces, thus leading to an under or overdose at the air cavity walls, along with lung-tissue interface or on the skin.,

The setup geometry for irradiation with the Elekta Unity is different with a conventional linac. The source-to-axis distance (SAD) for MR-Linac system is $143.5 \mathrm{~cm}$, instead of $100 \mathrm{~cm}$. The extended SAD results in a wider Multi-Leaf Collimator (MLC) leaf width at the isocenter plane, $7.15 \mathrm{~mm}$, compared to $5 \mathrm{~mm}$ leaf width of a similar MLC on a conventional linac. ${ }^{9,10}$ Another distinct feature of the Elekta Unity is that only the longitudinal couch movement is available for setting up the treatment isocenter in patient. Also, the Unity system does not allow collimator rotation. The MLC leaves move only in the cranio-caudal direction.

The existence of magnetic field and the above mentioned technical features with the Unity MR-Linac may have potential implications in the plan qualities compared to using a conventional linac for IMRT. Several studies have investigated the impact of a transversal $1.5 \mathrm{~T}$ high field magnetic field on dose distribution, especially at the tissue-air interfaces. ${ }^{8,10-13}$ However, these studies only involved a single tumor site and only focused on the influence of $1.5 \mathrm{~T}$ magnetic field. ${ }^{10-13}$ The purpose of this study is to investigate the feasibility and performance of 1.5 T MR-Linac for treating various tumor sites by comparing the dosimetry, treatment efficiency, and TPS usability with conventional linac based treatment planning.

\section{Materials and Methods}

\section{Treatment Planning}

A total of 30 patients ( 6 for brain, 6 for esophagus, 6 for lungs, 6 for vertebra and 6 for rectum) previously treated with a
Table 1. Differences in Irradiation Geometry Between a Conventional Versa HD Linac and the MR-Linac.

\begin{tabular}{lll}
\hline Device specifications & Versa HD & MR-Linac \\
\hline Static magnetic field & - & $1.5 \mathrm{~T}$ \\
Nominal beam energy & $6 \mathrm{MV} \mathrm{FFF}$ & $7 \mathrm{MV}$ FFF \\
Additional beam filtration & - & Cryostat \\
MLC leaf width at isocenter & $5.0 \mathrm{~mm}$ & $7.15 \mathrm{~mm}$ \\
MLC leaf travel direction & Arbitrary & Cranio-caudal \\
Source-to-axis distance & $100 \mathrm{~cm}$ & $143.5 \mathrm{~cm}$ \\
Isocenter position relative to patient & Variable & Fixed at bore center
\end{tabular}

MLC: Multi-Leaf Collimator, FFF: flattening filter free.

conventional linac (Elekta Versa $\mathrm{HD}^{\mathrm{TM}}$ ) at our institute were randomly selected from the clinical archive. Among the patients, 18 were men, 12 were women, and the median age was 51 years (range from 31 to 74). In a routine clinical procedure, a Computed Tomography (CT) simulation (Siemens Somatom $^{\mathrm{TM}}$, Munich, Germany) was acquired using $140-\mathrm{kVp}$ $\mathrm{X}$ rays and $0.3 \mathrm{~cm}$ uniform slice thickness. The target volume and organs at risk (OAR) were delineated by a radiation oncologist with specialty in that treatment site. The clinical plans were designed using Monaco (v5.11). The same planning CT and structure set were used for re-planning on Monaco (v5.40.01). The re-planning for simulated MR-Linac treatment was done by the same dosimetrists following the same institutional protocols.

The impact of magnetic field on dose calculation was taken into account in Monaco (v5.40.01) with the GPUMCD algorithm. ${ }^{14}$ The GPUMCD calculation would regress to the results of x-ray voxel Monte Carlo (XVMC) ${ }^{15}$ without magnetic field present for the clinical machine for the patients studies. The Unity beam model also accounted for transmission through the cryostat, the couch and receiver coils with a complicated set of attenuation parameters determined at the commissioning. The beam filtration and the nominal beam energy of the MR-Linac also differ from the $6 \mathrm{MV}$ photon beams with a Versa HD. Table 1 presents the main parameters of the 2 Elekta machines (Unity MR-Linac and Versa HD) for comparison.

The prescription doses were 60 Gy in 30 fractions for brain plans, 44 Gy in 20 fractions for esophagus plans, 45 Gy in 20 fractions for lung plans, $36 \mathrm{~Gy}$ in 10 fractions for vertebra plans and $50 \mathrm{~Gy}$ in 25 fractions for rectum plans. All the plans were designed with 5 coplanar fields except the brain cases, which were planned with 9 fields evenly spaced gantry angles. Since currently Elekta Unity only supports the step and shoot (ss) technique for IMRT, while the clinical plans used dynamic MLC (dMLC), for studying the delivery efficiency and TPS usability, reference ss-IMRT plans with Versa HD was generated for all cases. Based on our experience, the difference in the plan quality between dMLC-IMRT and ss-IMRT is negligible if the same beam setup and optimization parameters are used. The step-and-shoot plans for both MR-Linac and Versa HD were all limited to a maximum of 250 segments, while the plans for dMLC were limited to a maximum of 20 control 
Table 2. Calculation and Segmentation Parameters for the 3 Plan Groups.

\begin{tabular}{lccc}
\hline Plan parameters & MRL-IMRT & ss-IMRT & dMLC-IMRT \\
\hline Energy & 7 MV FFF & 6 MV FFF & 6 MV FFF \\
Algorithm & GPUMCD & XVMC & XVMC \\
IMRT technique & Step-and-shoot & Step-and-shoot & Dynamic MLC \\
Grid spacing (cm) & 0.3 & 0.3 & 0.3 \\
$\begin{array}{l}\text { Statistical uncertainty (\%) } \\
\quad \text { Per Control Point }\end{array}$ & 3 & 3 & 3 \\
$\begin{array}{l}\text { Minimum segment } \\
\quad \text { area (cm }{ }^{2} \text { ) }\end{array}$ & 2 & 2 & - \\
$\begin{array}{l}\text { Minimum segment } \\
\quad \text { width (cm) }\end{array}$ & 0.5 & 0.5 & 0.5 \\
$\begin{array}{l}\text { Minimum MU/segment } \\
\text { Maximum \# segments } \\
\quad \text { per plan }\end{array}$ & 4 & & - \\
$\begin{array}{l}\text { Maximum \# of Control } \\
\quad \text { Points per beam }\end{array}$ & 250 & 250 & - \\
\hline
\end{tabular}

points per beam. Other key parameters for the 3 groups plans for example nominal energy, dose calculation algorithm, grid spacing, statistical uncertainty, minimum segment area, minimum segment width, minimum MU per segment were presented in Table 2.

\section{Plan Evaluation and Comparison}

Plan evaluations were conducted by the attending physicians and clinical physicists originally assigned to the cases using the Dose-volume histograms (DVH) metrics based on the same institutional protocols. Parameters such as homogeneity index (HI) and conformality index (CI) were used to evaluate the targets dose homogeneity and conformality, while the mean dose $\left(\mathrm{D}_{\text {mean }}\right)$ and maximum point dose $\left(\mathrm{D}_{\max }\right)$ were used for OARs evaluation. The HI and CI are defined as ${ }^{16,17}$ :

$$
\begin{gathered}
\mathrm{HI}=\left(\mathrm{D}_{2 \%}-\mathrm{D}_{98 \%}\right) / \mathrm{D}_{50 \%} \\
\mathrm{CI}=\left(\mathrm{TV}_{\mathrm{PV}} \times \mathrm{TV}_{\mathrm{PV}}\right) /\left(\mathrm{V}_{\mathrm{PTV}} \times \mathrm{V}_{\mathrm{TV}}\right)
\end{gathered}
$$

where $\mathrm{D}_{2 \%}, \mathrm{D}_{98 \%}$ and $\mathrm{D}_{50 \%}$ represent the minimum dose covering $2 \%, 98 \%$ and $50 \%$ of the target volume, respectively. $V_{\mathrm{TV}}$ is the treatment volume of the body received the prescribed dose, $\mathrm{V}_{\mathrm{PTV}}$ is the volume of PTV, and $\mathrm{TV}_{\mathrm{PV}}$ is the target volume covered by the prescribed dose. The lower the $\mathrm{HI}$ value means the better the homogeneity. ${ }^{17} \mathrm{CI}$ is normally used to quantitatively measure the conformality of the dose distribution relative to the target volume, which denotes the ratio of reference dose received by targets and normal tissue. The CI value closer to 1 means a better conformality. ${ }^{18}$

For OARs, the $\mathrm{D}_{\max }$ and $\mathrm{D}_{\text {mean }}$ were used to evaluate for a serial organ, such as brain stem, spinal cord, optic chiasm, optic nerve, esophagus, bladder, small bowel and colon. While the $\mathrm{D}_{\text {mean }}$ and/or Vx (\% OARs volume receiving $\mathrm{x}$ Gy) were used to evaluate a parallel organ, such as lungs and femoral heads. For the skin dose evaluation, the interested volume is defined as the shell volume with the interior surface as a $5 \mathrm{~mm}$ contraction from the body contour. The $\mathrm{V}_{10}$ Gy of the unspecified normal tissue was calculated to evaluate the volume of the low dose region, which defined as body subtracted target volume. For assessing the impact of ERE to the tissue-lung surface, the maximum dose in a $5 \mathrm{~mm}$ thick layer inside of the lung surface was evaluated. ${ }^{11}$

The TPS usability is assessed by the time it took for generating an IMRT plan from initiating optimization stage 1 to the end of final dose calculation for each method. Additionally, the total number of MUs for each plan was also recorded to provide an estimate of the deliverability. ${ }^{19}$

All analyses were performed using IBM SPSS (v25) statistical software (IBM Corporation, Armonk, NY, USA). A Wilcoxon signed rank test was carried out and $P<0.05$ was considered statistically significant. Blinded reviews by the physicians and physicists were performed to assess the plan quality and delivery efficiency.

\section{Results}

\section{PTV Coverage and Dose to OARs}

Our institutional criteria for plan acceptance were met with all the 3 sets of plans (MRL-IMRT, ss-IMRT and dMLC-IMRT) for the 30 patients in this study. Similar PTV coverage with the prescribed dose was achieved, and the doses to OARs were also within the clinically accept limits. Figures $1-5$ showed the calculated DVH parameters for the plans of brain, esophagus, lung, vertebra, and rectum patients respectively. In Figures $1-5$, the differences in the investigated dosevolume metrics of various organs between the plans designed for the MRL-IMRT and ss-IMRT or AMLC-IMRT were presented with the box plot. The figure's horizontal axis shows the DVH metrics and evaluation parameters. Numerically positive differences mark an increase in the respective metric for the MR-Linac plans. If the DVHs are the CI and HI, the vertical axis shows absolute difference value, and if the DVHs are the $D_{\text {mean }}$ and $D_{\text {max }}$, the vertical axis shows absolute dose (Gy), and if the DVHs is Vx, the vertical axis shows percentage value. Furthermore, the Figure 5 only shows the PTV CI and HI for vertebra patients because no OARs were considered. Most parameters showed with minor differences among the 3 plan groups. The DVH parameters that showed statistically significant difference (at $P<0.05$ which was chosen) were denoted in the plots with asterisk.

For brain patients, the mean HI for MRL-IMRT, dMLCIMRT and ss-IMRT were $0.09 \pm 0.02,0.06 \pm 0.01$ and $0.07 \pm 0.01$, respectively. The differences between MRLIMRT and ss-IMRT and between MRL-IMRT and dMLCIMRT were found to be statistically significant, with $P=0.001$ and $P=0.019$ for the HI, respectively. In addition, there was a high point maximum dose in the lens with the MRL-IMRT plans, which was also acceptable (Lens $\mathrm{D}_{\max }$ : MRL-IMRT: $10.97 \pm 1.08$ Gy, dMLC-IMRT: $8.41 \pm 1.51$ Gy, ss-IMRT: $8.10 \pm 0.93 \mathrm{~Gy} ; P_{\text {MRL-IMRT vs dMLC-IMRT }}=$ $0.002, P_{\text {MRL-IMRT vs ss-IMRT }}=0.003$ ) (Figure 1 ). For esophagus plans, the result in the investigated dose-volume metrics of 


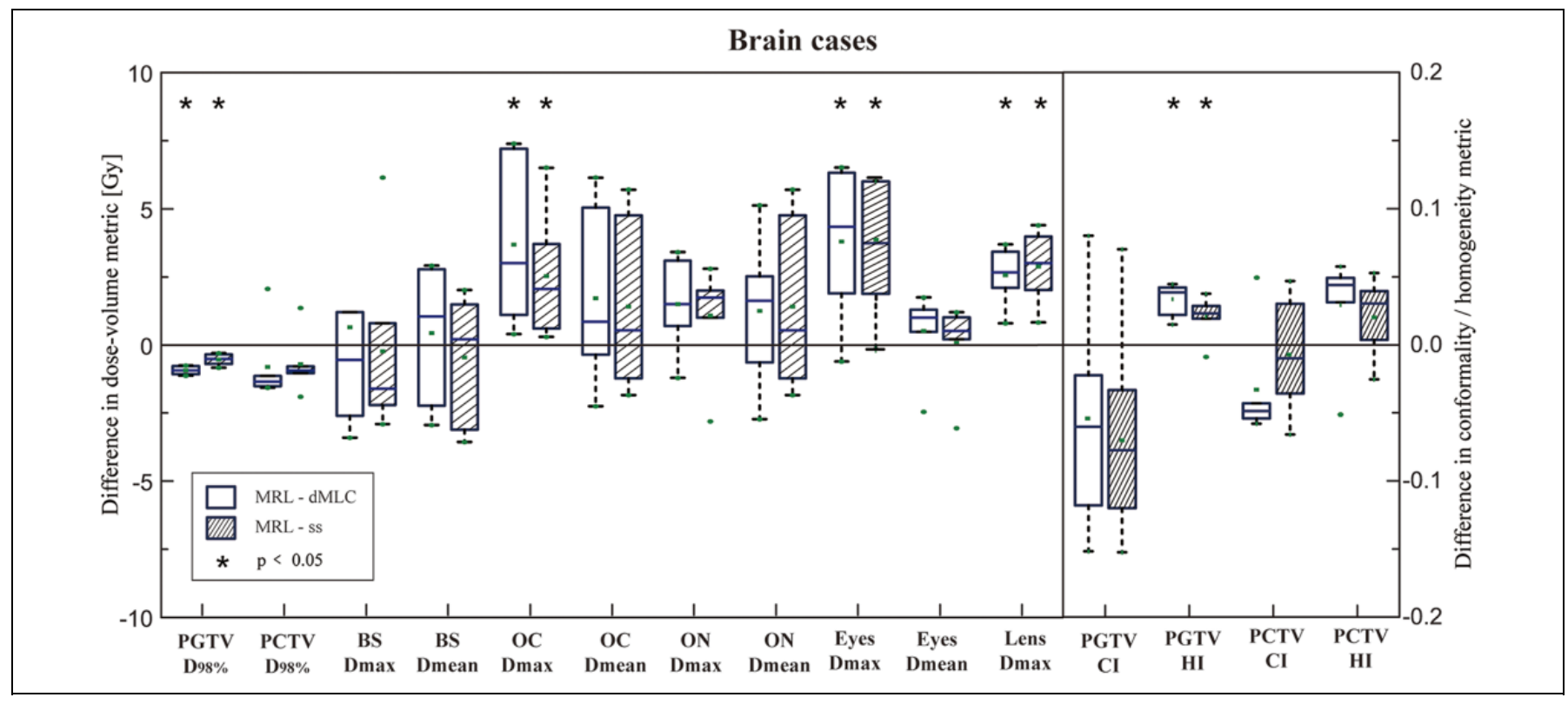

Figure 1. Differences in the investigated dose-volume metrics between the plans generated for the conventional linac with either dynamic MLC (dMLC) or step and shoot (ss) delivery technique and the MR-linac (MRL) for brain cases. positive differences mark an increase in the respective metric for the MR-linac plans. the boxes mark the 5th and 95th percentiles, the band marks the median, the dot marks the outlying values. A * indicates a significance of $P<0.05$. BS: brain stem, OC: optic chiasma, ON: optic nerve, CI: conformality index, HI: homogeneity index.

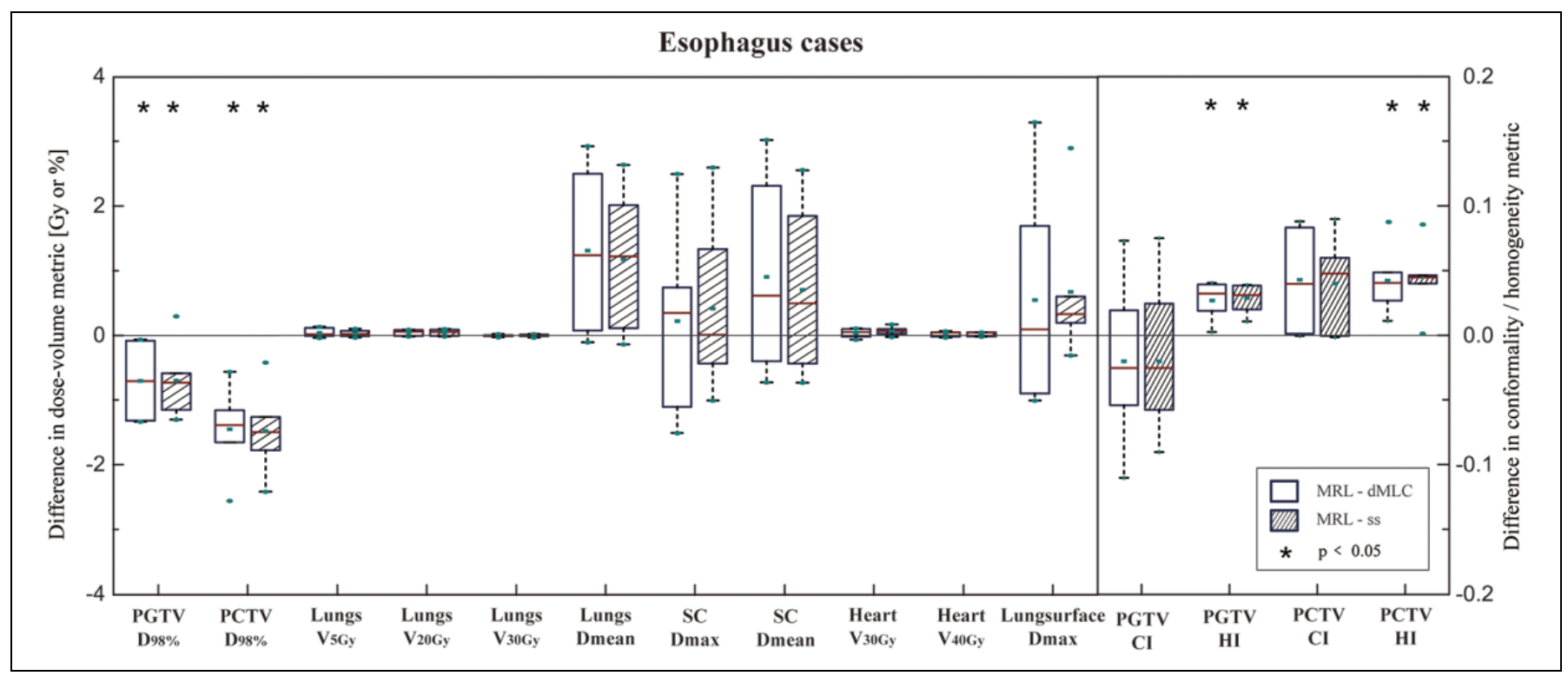

Figure 2. Differences in the investigated dose-volume metrics between the plans generated for the conventional linac with either dynamic MLC (dMLC) or step and shoot (ss) delivery technique and the MR-linac (MRL) for esophagus cases. positive differences mark an increase in the respective metric for the MR-linac plans. the boxes mark the 5th and 95th percentiles, the band marks the median, the dot marks the outlying values. A * indicates a significance of $P<0.05$. SC: spinal cord, $\mathrm{CI}$ : conformality index, HI: homogeneity index.

various organs showed that only PTV homogeneity has statistical differences (Figure 2). The lung, rectum and vertebra plans showed minor significant statistical differences between MRL-IMRT and ss-IMRT and between MRL-IMRT and dMLC-IMRT (Figures 3-5).
Table 3 shows the maximum and mean dose of the skin and $\mathrm{V}_{10 \text { Gy }}$ of the unspecified normal tissue. The MRL-IMRT plans had higher dose to the skin roughly by $6 \%$ on average, and greater low dose volumes roughly by $8 \%$ on average. The differences in the skin dose maximum were statistically 


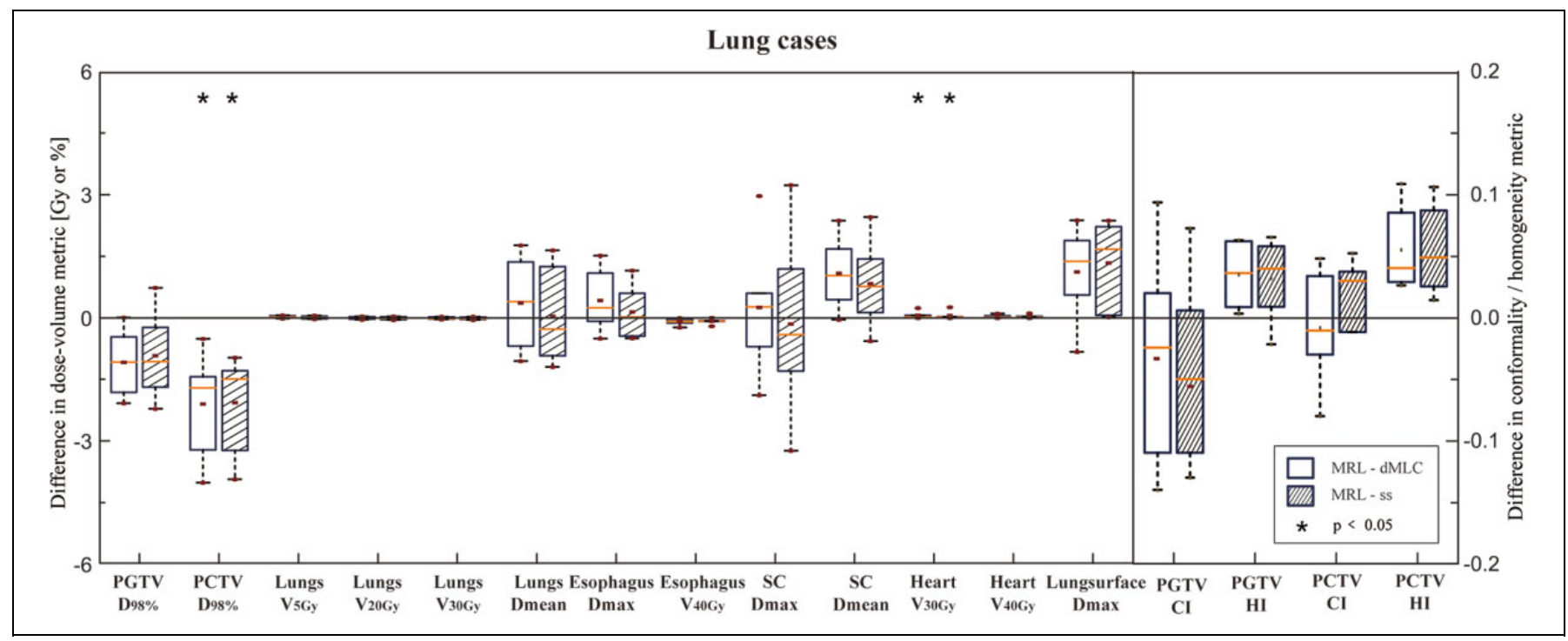

Figure 3. Differences in the investigated dose-volume metrics between the plans generated for the conventional linac with either dynamic MLC (dMLC) or step and shoot (ss) delivery technique and the MR-linac (MRL) for lung cases. positive differences mark an increase in the respective metric for the MR-linac plans. the boxes mark the 5th and 95th percentiles, the band marks the median, the dot marks the outlying values. A * indicates a significance of $P<0.05$. SC: spinal cord, CI: conformality index, HI: homogeneity index.

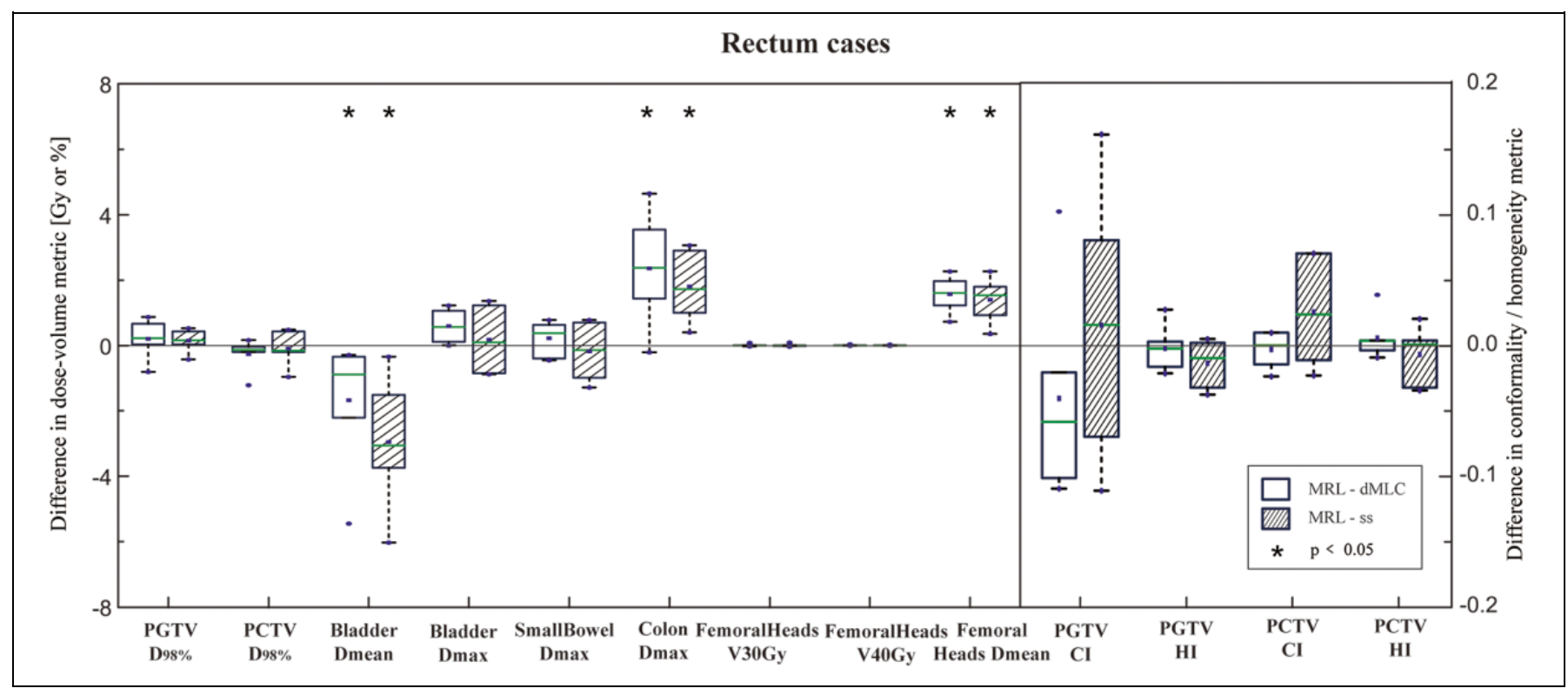

Figure 4. Differences in the investigated dose-volume metrics between the plans generated for the conventional linac with either dynamic MLC (dMLC) or step and shoot (ss) delivery technique and the MR-linac (MRL) for rectum cases. positive differences mark an increase in the respective metric for the MR-linac plans. the boxes mark the 5th and 95th percentiles, the band marks the median, the dot marks the outlying values. A * indicates a significance of $P<0.05$. CI: conformality index, HI: homogeneity index.

significant, with $P=0.037$ between MRL-IMRT and ss-IMRT, and $P=0.018$ between MRL-IMRT and dMLC-IMRT, respectively. Likewise, the low dose volume increases from ss-IMRT and dMLC-IMRT had $P<0.001$, respectively. The subtractions of the dose distributions were also analyzed in order to identify relevant differences, which are not covered by the chosen parameters. Within this analysis we inspected the difference in dose distributions as shown in Figure 6 (the differences between
MRL-IMRT and dMLC-IMRT) and Figure 7 (the differences between MRL-IMRT and ss-IMRT) for a typical esophagus case. While dose deviations within the boost volume are marginal, more pronounced differences are observed outside the target volume at the cranial and caudal field margins in Figure 6 $\mathrm{a} 3, \mathrm{~b} 3$ and Figure $7 \mathrm{a} 3, \mathrm{~b} 3$. The DVH plot for this case is shown in Figure 8. Although the difference in minimum dose to PTVs is large, MRL-IMRT plan meets the clinical requirements of 


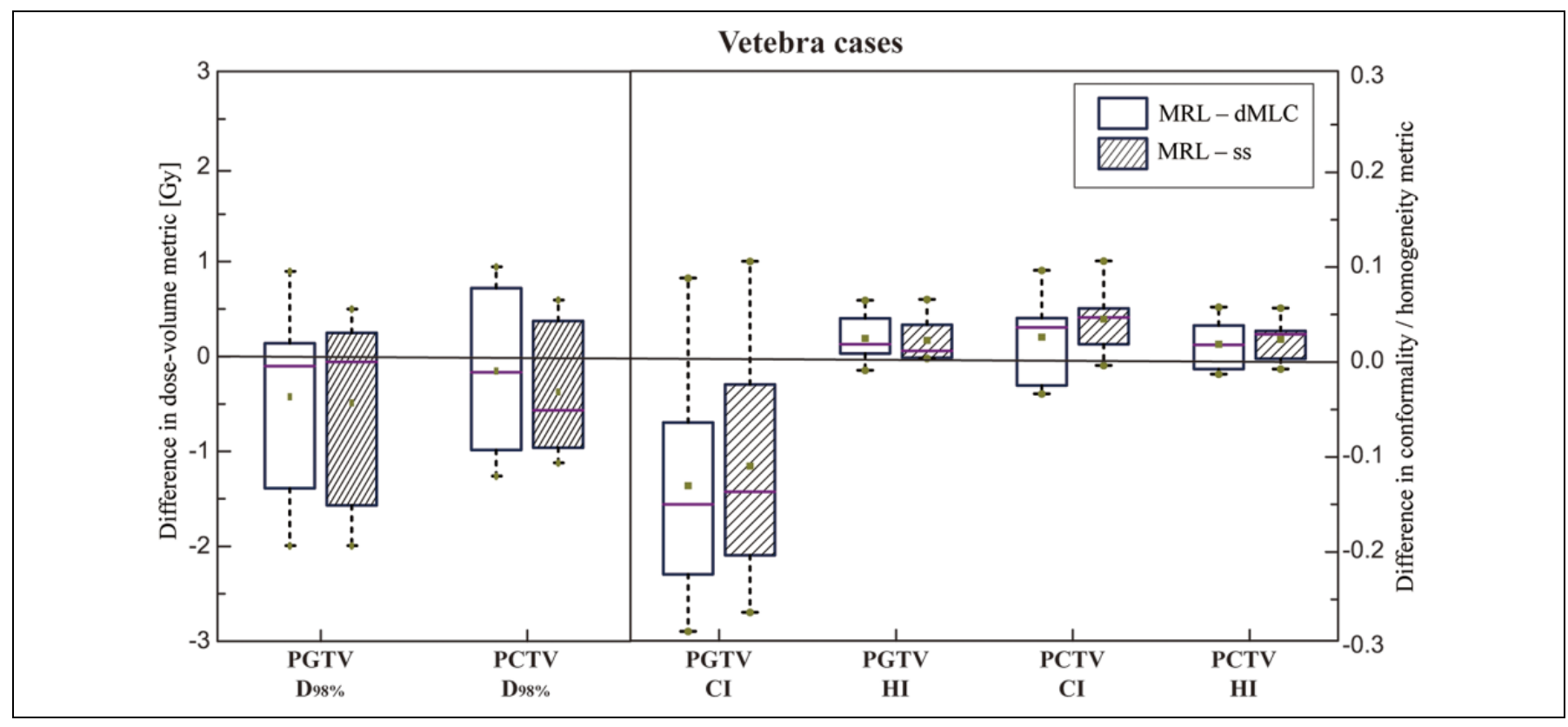

Figure 5. Differences in the investigated dose-volume metrics between the plans generated for the conventional linac with either dynamic MLC (dMLC) or step and shoot (ss) delivery technique and the MR-linac (MRL) for vetebra cases. positive differences mark an increase in the respective metric for the MR-linac plans. the boxes mark the 5th and 95th percentiles, the band marks the median, the dot marks the outlying values. CI: conformality index, HI: homogeneity index.

Table 3. Comparison of Dose to Skin and Normal Tissue in All 3 Radiotherapy Plan Groups.

\begin{tabular}{llrrrrr}
\hline & & MRL-IMRT & ss-IMRT & dMLC-IMRT & $P_{\text {MRL vs Ss }}$ & $P_{\text {MRL vs dMLC }}$ \\
\hline \multirow{2}{*}{ Skin } & $\mathrm{D}_{\max }(\mathrm{Gy})$ & $40.79 \pm 9.68$ & $39.64 \pm 9.51$ & $39.62 \pm 9.39$ & 0.037 & 0.018 \\
& $\mathrm{D}_{\text {mean }}(\mathrm{Gy})$ & $4.63 \pm 2.41$ & $4.04 \pm 2.20$ & $3.75 \pm 1.95$ & $<0.001$ & $<0.001$ \\
NT & $\mathrm{V}_{10 \text { Gy }}(\%)$ & $24.45 \pm 12.09$ & $22.69 \pm 11.30$ & $22.07 \pm 10.95$ & $<0.001$ & $<0.001$ \\
\hline
\end{tabular}

target coverage. Which demonstrates that the MR-Linac plan was clinically acceptable.

\section{Optimization Time and Delivery Efficiency}

The average MUs and optimization time for each plan group are shown in Table 4. The optimization time for the MRLIMRT, ss-IMRT and dMLC-IMRT plans were $6.4 \pm 3.1 \mathrm{~min}$, $11.1 \pm 5 \mathrm{~min}, 17.9 \pm 6 \mathrm{~min}$, respectively. MRL-IMRT reduced the average optimization time by $42.3 \%$ and $64.2 \%$ compared with ss-IMRT and dMLC-IMRT, with $P<0.001$, respectively. The total MUs for the MRL-IMRT, ss-IMRT and dMLC-IMRT plans were 714.7 $\pm 271.51,550.42 \pm 155.58$, $580.42 \pm 116.7$, respectively. Significant difference of the MUs between MRL-IMRT and ss-IMRT was observed $(P=0.001)$, while the difference between MRL-IMRT and dMLC-IMRT group was not statistically significant $(P=0.091)$.

\section{Discussion}

This study included brain, esophagus, lung, vertebra and rectum patients previously treated with a conventional linear accelerator. The PTV size under the limit was the only inclusion/exclusion criteria for patient selection, since the Elekta 1.5 T MR-Linac allows the maximum field size of $22 \mathrm{~cm}$ in the superior-inferior direction at isocenter. ${ }^{20}$ For the patient cohort, the longitudinal length of the PTV was $12.3 \pm$ $4.0 \mathrm{~cm}$, and the volume of the PTV was $500.5 \pm 309.0 \mathrm{~cm} .^{3}$ Several published studies have shown that the Monaco is capable of producing clinically acceptable plans for the $1.5 \mathrm{~T} \mathrm{MR}$ Linac on a few tumor sites. ${ }^{10,12,13,21}$ Others have evaluated the quality of IMRT plans using a ${ }^{60} \mathrm{Co}$ MRI guided radiation therapy system. ${ }^{22,23}$ Our study shows that it is feasible to use the 1.5 T MR-Linac to treat multiple tumor sites with IMRT based on clinically validated protocols and comparative dosimetry. This means that the MR-Linac can be used to treat future patents with these tumor sites for exploring the potential advantages of more accurate targeting in treatment delivery.

MRL-IMRT plans have been shown to meet the clinical requirements of target coverage and OARs sparing successfully for multiple tumor sites in our study. However, there were small differences in the target dose homogeneity and conformality, and the OAR dose, noticeably in the brain plans. In contrast with our study, Nachbar et al and van de Schoot et al evaluated the treatment plans quality between 1.5 T MR-Linac and a conventional linac for esophageal, rectal and prostate cancer. ${ }^{10,24}$ Their 

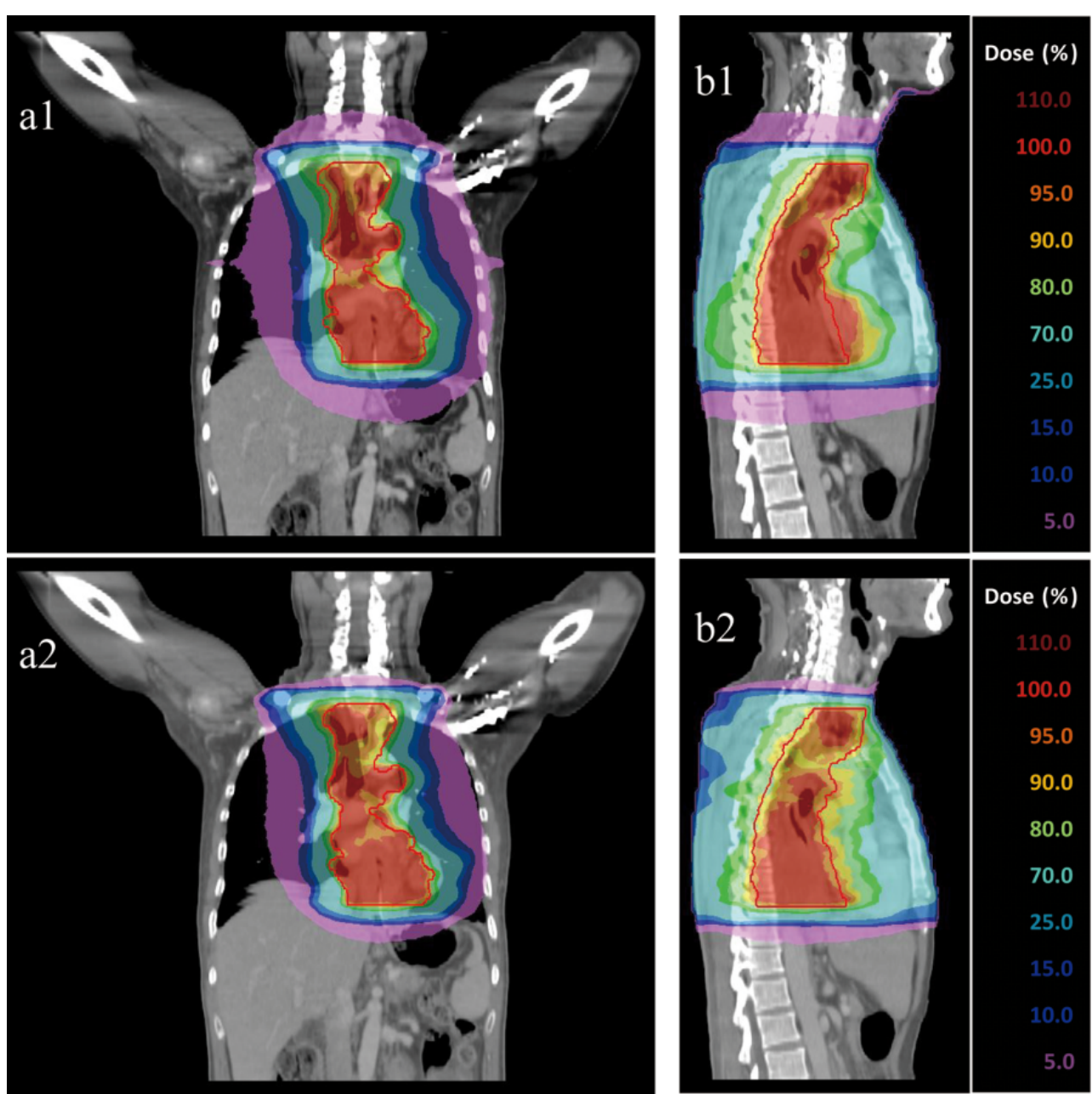

Dose (\%)

110.0

100.0

95.0

90.0

80.0

70.0

25.0

15.0

10.0

5.0
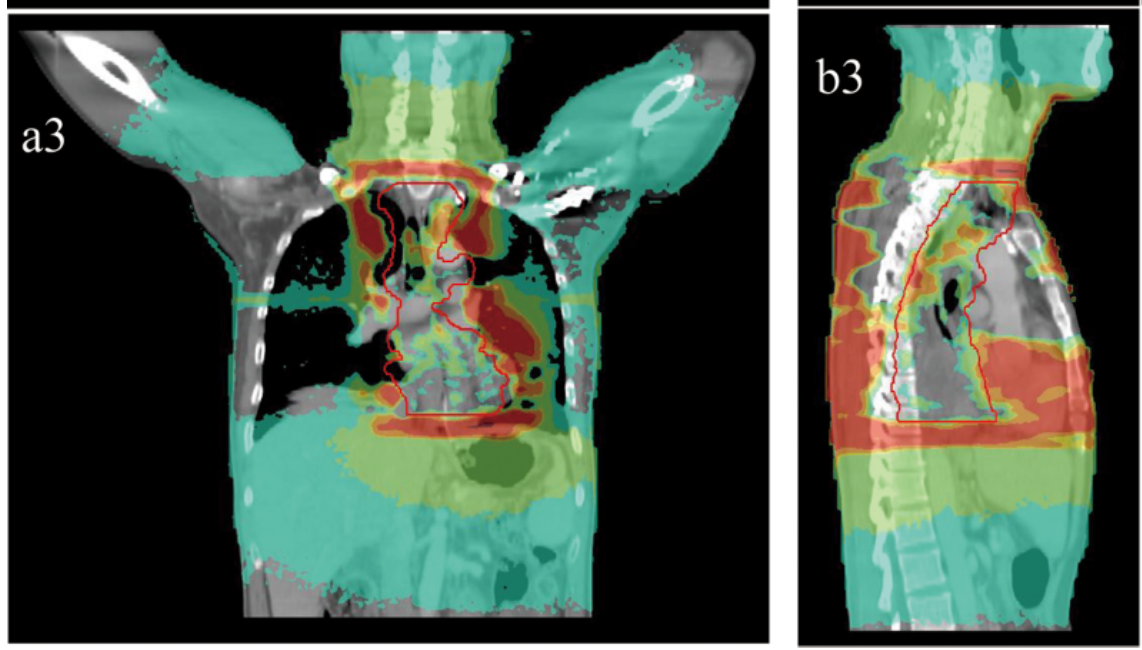

Dose(\%)

30.0

15.0

10.0

5.0

2.0

1.0

0.0

Figure 6. Sagittal and coronal views of the target volume and dose distributions for the MR-linac plan (a1 and b1), the conventional linac based dynamic MLC plan (a2 and b2) and a subtraction of the dose distribution between both plans (a3 and b3 MRL-IMRT-dMLC-IMRT). light red marks PTV.

results are similar to our findings. The plan quality reflected by target volume conformality and homogeneity is closely related to the complexity of target volume geometry in relation with adjacent OARs, the delivery equipment and technique, and the optimization algorithm. ${ }^{17}$ The worse homogeneity and higher lens and eyes dose for MR-Linac plans was primarily the result of increased source to isocenter distance, effectively increased MLC leaf width, and increased penumbra. In previous studies, 


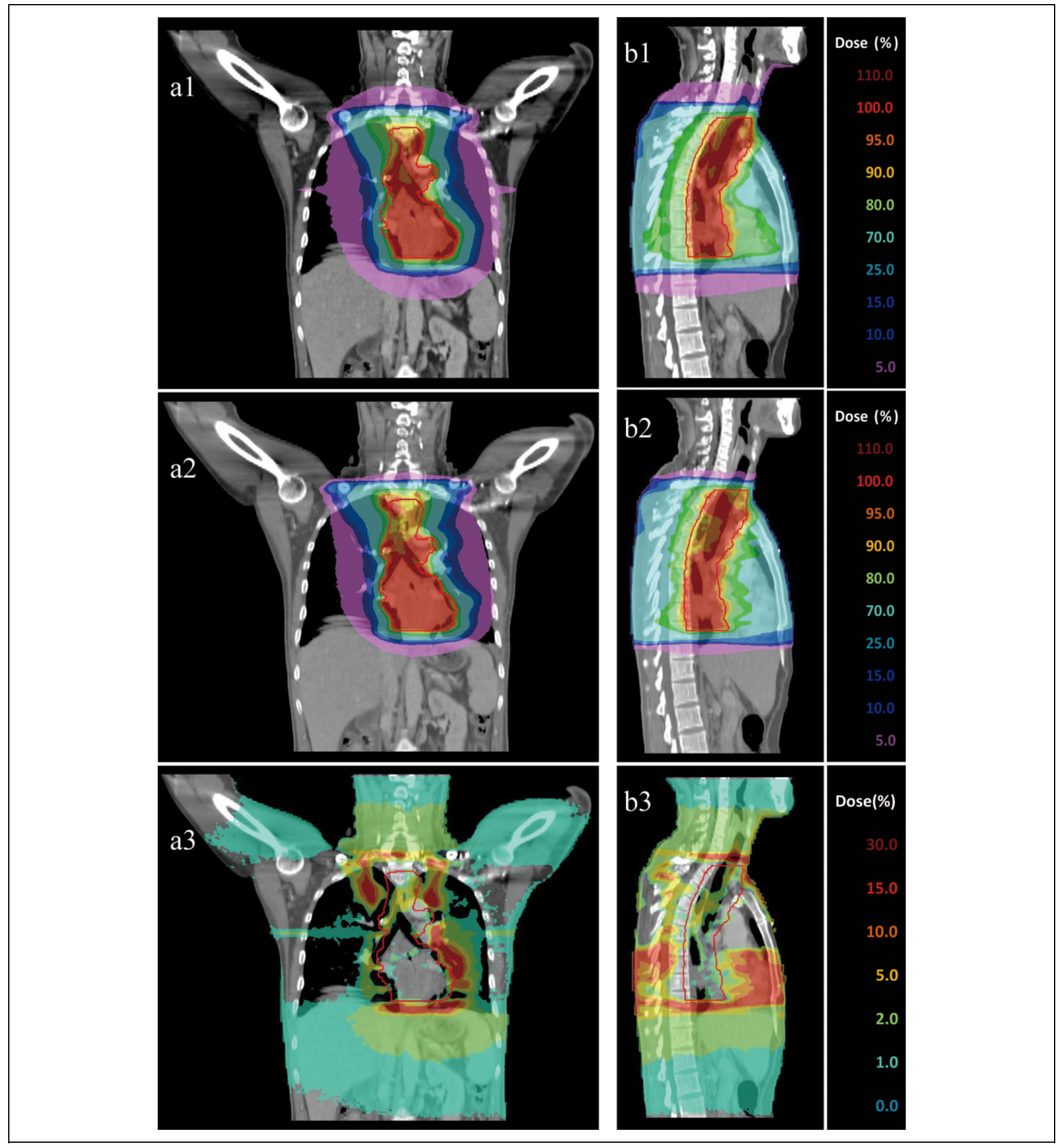

Figure 7. Sagittal and coronal views of the target volume and dose distributions for the MR-linac plan (a1 and b1), the conventional linac based step and shoot plan ( $\mathbf{2} 2$ and b2) and a subtraction of the dose distribution between both plans (a3 and b3 MRL-IMRT-ss-IMRT). light red marks PTV.

the authors have demonstrated that the smaller MLC leaf width can provide better dosimetric outcomes. ${ }^{25,26}$ In addition, the MLC leaves can travel only in the cranio-caudal direction and the conventional linac MLC leaf moves in the arbitrary direction, thus the field portals can be less than optimal to conform with target. Apart from that, the MR-Linac isocenter position relative to patient is fixed at bore center, which might not always be ideal. These MR-Linac features were considered relevant to 
have an impact to the plan quality. For brain cases, although the literature reported that the brain IMRT can be planned using noncoplanar fields to avoid the eyes and lens, ${ }^{27}$ the noncoplanar IMRT can't be generated with MR-Linac because the treatment couch is fixed in left-right direction. In our study, we mainly focus on the differences in plan quality between MR-Linac and standard linac due to the differences in machine characteristics and magnetic field. To make the comparison fairly and reduce the variability, coplanar IMRT planning was adopted. However, the individualized beam angles can be used to avoid eyes and lens and likely appear a higher conformity and marginally better OAR sparing in comparison of 9 equidistant beam angle used in this study. Additionally, we used the similar IMRT constrains in optimization process for different delivery techniques in our study. It appears likely that with a stricter prescription equal doses between the 2 devices can be achieved.

MR-guided dose delivery is inevitably affected by the presence of the permanent magnetic field. The Lorentz force influences the trajectory of secondary electrons. The produced secondary electrons may return to the tissue surface at the air-tissue interface, as so-called ERE. ${ }^{21}$ Due to this effect, hot spots and cold spots can arise around the air cavities and skin. The ERE takes effect at air cavities if the cavity is larger than once or twice the electron trajectory radius in air. Dose levels before such cavities will be increased. After such cavities, full buildup has to be re-established, since no electrons can pass the cavity. In these regions dose levels are lower. ${ }^{8}$ This effect is of major concern where there is air volume present in or near the

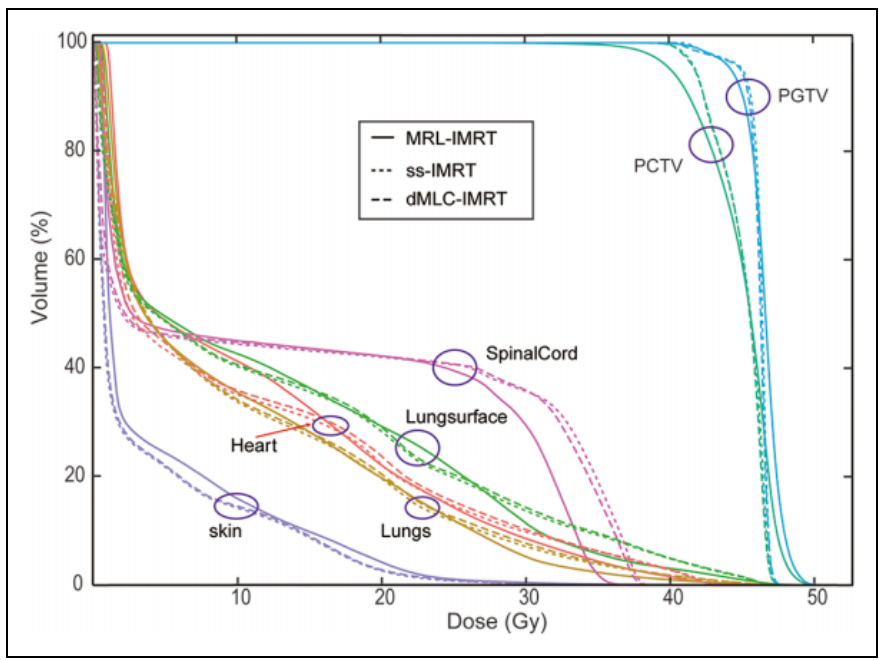

Figure 8. Dose-volume histogram for PTVs and OARs in the typical esophagus case. target volume, such as in a head and neck case, and in lung or pelvic region. ${ }^{21}$ In our study, the ERE apparently did not induce remarkable dose effects around air cavities in patient body of brain, lung, esophagus and pelvic cases, perhaps the multiple beam directions in IMRT plans largely neutralized the dose perturbation by the magnetic field. The maximum dose at the lung surface, defined as a $5 \mathrm{~mm}$ inner layer around the lung contours, was not significantly different with the conventional linac plans. This is consistent with the results reported by previous studies, such as that by Chen et al. ${ }^{12}$ The irregular shape of air cavity inside the human body can be another reason why the magnetic field effect is not as significant as that seen in the phantom study. Furthermore, the inclusion of the effect of the transverse magnetic field in IMRT plan optimization can significantly reduce, or even completely remove, the dose effects on the air-tissue or lung-tissue interfaces inside the body. However, the observed increases in skin dose for the MR-Linac plans were expected (Table 3), and consistent with other published studies. ${ }^{10-12,21}$ This was clearly the consequence of ERE under the $1.5 \mathrm{~T}$ magnetic field. Firstly, the point spread kernel becomes asymmetrical which results in a reduced build-up distance. Then the strong dose increase is observed due to secondary electrons that are forced back into the tissue by the Lorentz force at the exit dose if the beam exits into air. ${ }^{28}$ Both of the 2 aspects contribute to the increase of skin doses.

Furthermore, a significant increase of the low dose region in the normal tissues was also observed in our study. The similar findings were also observed in Agustinus et al study. ${ }^{24}$ The dosedifference maps showed the higher doses in a region of approximately $3 \mathrm{~cm}$ at the superior and inferior edges of the target volume with the MR-Linac. This is largely due to the fixed MLC leaf direction and no Jaw in cranio-caudal direction. And this observation is also possibly associated with the increased source to isocenter distance and consequently a different divergence of the photon beams. Interestingly, we observed the dose increase in chin for MRL-IMRT (Figures 6 and 7). The effect could possibly be associated with the electron streaming effect (ESE) that transverse magnetic field sweeps the contaminant electrons away from the radiation beam and traveling along the magnetic field direction. ${ }^{29}$ Hackett $e t a l^{30}$ observed that for large fields, the spiraling contaminant electrons (SCE) dose was in the same order of magnitude as that from scattered and leakage photons, and the dose for both SCE and scattered photons decreased rapidly with decreasing beam size and increasing distance from the beam edge.

This study also demonstrated that the planning efficiency of MR-Linac IMRT plan was superior to conventional linac based IMRT plans. The average optimization time for MRL-IMRT

Table 4. Comparison of Efficiency for All 3 Plan Groups.

\begin{tabular}{lcccrc}
\hline & MRL-IMRT & ss-IMRT & dMLC-IMRT & $P_{\text {MRL vs Ss }}$ & $P_{\text {MRL vs dMLC }}$ \\
\hline $\begin{array}{l}\text { Optimization } \\
\text { Time (min) }\end{array}$ & $6.4 \pm 3.1$ & $11.1 \pm 5.0$ & $17.9 \pm 6.0$ & $<0.001$ \\
MUs & $714.7 \pm 271.51$ & $550.42 \pm 155.58$ & $580.42 \pm 116.7$ & 0.001 \\
\hline
\end{tabular}


plan was reduced by $42.3 \%$ and $64.2 \%$ compared with ss-IMRT and dMLC-IMRT, respectively. This is mainly owing to that the MR-Linac specific TPS Monaco (v5.40.01) employs a much fast dose engine based on a graphic processing unit (GPU)-based Monte Carlo dose calculation platform (GPUMCD). ${ }^{12,14}$

The number of MUs has been used as a surrogate measure for plan complexity assessment. ${ }^{11}$ In this study, the average number of MUs for the MRL-IMRT plans was nearly $30 \%$ higher than the ss-IMRT plans, but no statistically significant difference was observed for the dMLC-IMRT plans. The dose rate for Unity MR-Linac is $425-450 \mathrm{MU} / \mathrm{min}$, which is less than that of a conventional linac (generally $600-1400 \mathrm{MU} / \mathrm{min}$ ). It has been reported that the beam-on time would decrease by 10 to 40 seconds for every $100 \mathrm{MU} / \mathrm{min}$ increase of dose rate. ${ }^{31}$ Therefore, it is expected that the delivery time for MR-Linac is longer compared with the conventional linac. Currently, only the step-and-shoot delivery technique can be used for MR-Linac system, while the dynamic MLC delivery technique has been used with conventional linacs in our clinical practice. The step-and-shoot technique delivery time is also longer than dynamic MLC technique. Dynamic MLC delivery in the future will improve the delivery efficiency for Elekta Unity.

\section{Conclusions}

It was feasible to create clinically acceptable IMRT plans with a 1.5 T MR-Linac system for multiple tumor sites. With the GPUMCD dose engine, the planning efficiency of MRL-IMRT was improved with much reduced optimization time. Given the differences in machine characteristics, some minor differences in plan quality were found between MR-Linac plans and current clinical practice and this should be considered in clinical practice. In general, the results support the clinical introduction of treatment planning for MR-linac, and a baseline has been established for evaluation of the effect of future adaptative planning strategies based on repeated multiparametric imaging.

\section{Acknowledgments}

The authors wish to thank the colleagues who kindly agreed to share their data and provide help: Shaoxiong Wu, Meiling Deng, Mengzhong Liu, Hui Liu, Yuanhong Gao, Zhifan Zeng, Jinhan Zhu.

\section{Author Contribution}

Shouliang Ding, MS, Yongbao Li, PhD, Hongdong Liu, $\mathrm{PhD}$ are contributed equally to this work.

\section{Declaration of Conflicting Interests}

The author(s) declared no potential conflicts of interest with respect to the research, authorship, and/or publication of this article.

\section{Ethical Statement}

Our study was approved by Sun Yat-sen University Cancer Center Ethics Committee (approval no. C2019-006-01). All patients provided written informed consent prior to enrollment in the study.

\section{Funding}

The author(s) received no financial support for the research, authorship, and/or publication of this article: This work was supported by National Natural Science Foundation of China (No. 11805292); Natural Science Foundation of Guangdong, China (No. 2018A030 3100020)

\section{ORCID iD}

Xiaoyan Huang (D) https://orcid.org/0000-0002-7811-3350

\section{References}

1. Tijssen RHN, Philippens MEP, Paulson ES, et al. MRI commissioning of 1.5T MR-linac systems - a multi-institutional study. Radiother Oncol. 2019;132:114-120.

2. Lagendijk JJ, Raaymakers BW, Van den Berg CA, Moerland MA, Philippens ME, van Vulpen M. MR guidance in radiotherapy. Phys Med Biol. 2014;59(21):R349-R369.

3. Lagendijk JJ, Raaymakers BW, Raaijmakers AJ, et al. MRI/linac integration. Radiother Oncol. 2008;86(1):25-29.

4. Noel CE, Parikh PJ, Spencer CR, et al. Comparison of onboard low-field magnetic resonance imaging versus onboard computed tomography for anatomy visualization in radiotherapy. Acta Oncol. 2015;54(9):1474-1482.

5. Fischer-Valuck BW, Henke L, Green O, et al. Two-and-a-halfyear clinical experience with the world's first magnetic resonance image guided radiation therapy system. Adv Radiat Oncol. 2017; 2(3):485-493.

6. Cunningham JM, Barberi EA, Miller J, Kim JP, Glide-Hurst CK. Development and evaluation of a novel MR-compatible pelvic end-to-end phantom. J Appl Clin Med Phys. 2019;20(1):265-275.

7. Raaymakers BW, Raaijmakers AJ, Kotte AN, Jette D, Lagendijk JJ. Integrating a MRI scanner with a $6 \mathrm{MV}$ radiotherapy accelerator: dose deposition in a transverse magnetic field. Phys Med Biol. 2004;49(17):4109-4118.

8. Raaijmakers AJ, Raaymakers BW, Lagendijk JJ. Integrating a MRI scanner with a $6 \mathrm{MV}$ radiotherapy accelerator: dose increase at tissue-air interfaces in a lateral magnetic field due to returning electrons. Phys Med Biol. 2005;50(7):1363-1376.

9. Bainbridge HE, Menten MJ, Fast MF, Nill S, Oelfke U, McDonald F. Treating locally advanced lung cancer with a $1.5 \mathrm{~T}$ MRlinac-effects of the magnetic field and irradiation geometry on conventionally fractionated and isotoxic dose-escalated radiotherapy. Radiother Oncol. 2017;125(2):280-285.

10. Nachbar M, Monnich D, Kalwa P, Zips D, Thorwarth D, Gani C. Comparison of treatment plans for a high-field MRI-linac and a conventional linac for esophageal cancer. Strahlenther Onkol. 2019;195(4):327-334.

11. Chuter R, van Herk M, Akhiat $\mathrm{H}$, et al. Comparison of intensity modulated radiotherapy plan optimisation methods for a $1.5 \mathrm{~T}$ MR-linac. J Appl Clin Med Phys. 2019;20(1):43-49.

12. Chen X, Prior P, Chen GP, Schultz CJ, Li XA. Technical note: dose effects of $1.5 \mathrm{~T}$ transverse magnetic field on tissue interfaces in MRI-guided radiotherapy. Med Phys. 2016;43(8):4797.

13. Menten MJ, Fast MF, Nill S, Kamerling CP, McDonald F, Oelfke U. Lung stereotactic body radiotherapy with an MR-linac- 
quantifying the impact of the magnetic field and real-time tumor tracking. Radiother Oncol. 2016;119(3):461-466.

14. Hissoiny S, Raaijmakers AJ, Ozell B, Despres P, Raaymakers BW. Fast dose calculation in magnetic fields with GPUMCD. Phys Med Biol. 2011;56(16):5119-5129.

15. Roche M, Crane R, Powers M, Crabtree T. Agility MLC transmission optimization in the Monaco treatment planning system. J Appl Clin Med Phys. 2018;19(5):473-482.

16. 3. Special considerations regarding absorbed-dose and dosevolume prescribing and reporting in IMRT. J ICRU. 2010; 10(1):27-40.

17. Ning ZH, Mu JM, Jin JX, et al. Single arc volumetric-modulated arc therapy is sufficient for nasopharyngeal carcinoma: a dosimetric comparison with dual arc VMAT and dynamic MLC and step-and-shoot intensity-modulated radiotherapy. Radiat Oncol. 2013;8:237.

18. Feuvret L, Noel G, Mazeron JJ, Bey P. Conformity index: a review. Int J Radiat Oncol Biol Phys. 2006;64(2):333-342.

19. Craft D, Suss P, Bortfeld T. The tradeoff between treatment plan quality and required number of monitor units in intensitymodulated radiotherapy. Int J Radiat Oncol Biol Phys. 2007; 67(5):1596-1605.

20. Chuter RW, Whitehurst P, Choudhury A, van Herk M, McWilliam A. Technical note: investigating the impact of field size on patient selection for the 1.5 T MR-linac. Med Phys. 2017;44(11): 5667-5671.

21. Uilkema S, van der Heide U, Sonke JJ, Moreau M, van Triest B, Nijkamp JA. 1.5T transverse magnetic field in radiotherapy of rectal cancer: impact on the dose distribution. Med Phys. 2015; 42(12):7182-7189.

22. Merna C, Rwigema JC, Cao M, et al. A treatment planning comparison between modulated tri-cobalt-60 teletherapy and linear accelerator-based stereotactic body radiotherapy for central early-stage non-small cell lung cancer. Med Dosim. 2016;41(1): 87-91.
23. Wooten HO, Green O, Yang M, et al. Quality of intensity modulated radiation therapy treatment plans using a (6)(0)co magnetic resonance image guidance radiation therapy system. Int J Radiat Oncol Biol Phys. 2015;92(4):771-778.

24. van de Schoot AJ, van den Wollenberg W, Carbaat C, et al. Evaluation of plan quality in radiotherapy planning with an MR-linac. Physics and Imaging in Radiation Oncology. 2019; 10:19-24.

25. Hong CS, Ju SG, Kim M, et al. Dosimetric effects of multileaf collimator leaf width on intensity-modulated radiotherapy for head and neck cancer. Med Phys. 2014;41(2):021712.

26. Zwicker F, Hauswald H, Nill S, et al. New multileaf collimator with a leaf width of $5 \mathrm{~mm}$ improves plan quality compared to $10 \mathrm{~mm}$ in step-and-shoot IMRT of HNC using integrated boost procedure. Strahlenther Onkol. 2010;186(6):334-343.

27. Panet-Raymond V, Ansbacher W, Zavgorodni S, et al. Coplanar versus noncoplanar intensity-modulated radiation therapy (IMRT) and volumetric-modulated arc therapy (VMAT) treatment planning for fronto-temporal high-grade glioma. J Appl Clin Med Phys. 2012;13(4):3826.

28. Raaijmakers AJ, Raaymakers BW, Lagendijk JJ. Magnetic-fieldinduced dose effects in MR-guided radiotherapy systems: dependence on the magnetic field strength. Phys Med Biol. 2008;53(4): 909-923.

29. Malkov VN, Hackett SL, Wolthaus JWH, Raaymakers BW, van Asselen B. Monte carlo simulations of out-of-field surface doses due to the electron streaming effect in orthogonal magnetic fields. Phys Med Biol. 2019;64(11):115029.

30. Hackett SL, van Asselen B, Wolthaus JWH, et al. Spiraling contaminant electrons increase doses to surfaces outside the photon beam of an MRI-linac with a perpendicular magnetic field. Phys Med Biol. 2018;63(9):095001.

31. Ghasroddashti E, Smith WL, Quirk S, Kirkby C. Clinical consequences of changing the sliding window IMRT dose rate. J Appl Clin Med Phys. 2012;13(4):3810. 